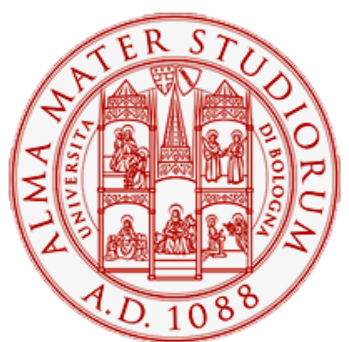

Alma Mater Studiorum - Università di Bologna DEPARTMENT OF ECONOMICS

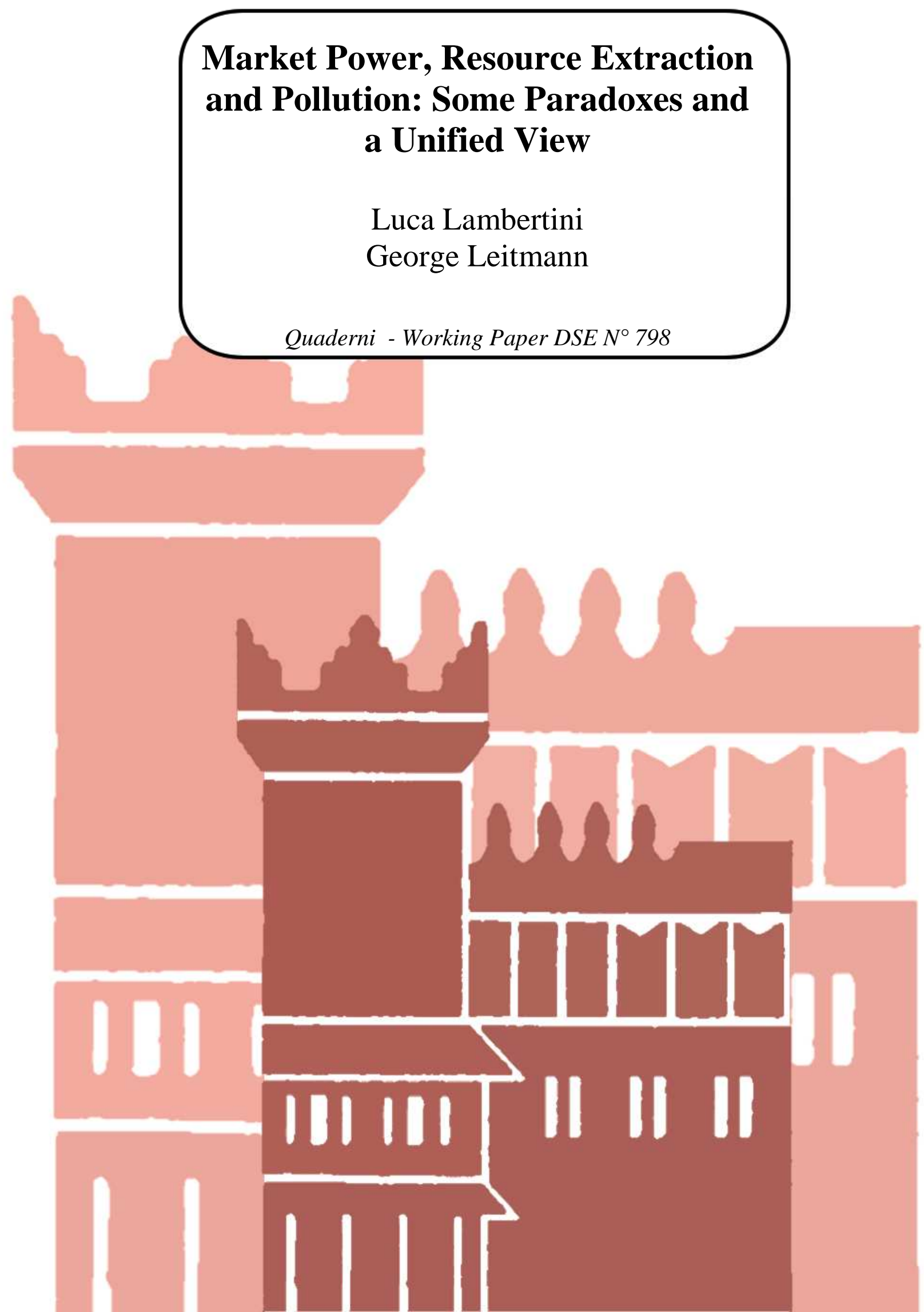




\title{
Market Power, Resource Extraction and Pollution: Some Paradoxes and a Unified View*
}

\author{
Luca Lambertini" ${ }^{\#}$ and George Leitmann ${ }^{\S}$ \\ \# Department of Economics, University of Bologna \\ Strada Maggiore 45, 40125 Bologna, Italy \\ fax +39-051-2092664; luca.lambertini@unibo.it \\ $\S$ College of Engineering, University of California at Berkeley \\ Berkeley CA 94720, USA; gleit@berkeley.edu
}

November 29, 2011

\begin{abstract}
We adopt a stepwise approach to the analysis of a dynamic oligopoly game in which production makes use of a natural resource and pollutes the environment, starting with simple models where firms' output is not a function of the natural resource to end up with a full-fledged model in which (i) the resource is explicitly considered as an input of production and (ii) the natural resource and pollution interact via the respective state equations. This allows us to show that the relationship between the welfare properties of the economic system and the intensity of competition is sensitive to the degree of accuracy with which the model is constructed.
\end{abstract}

JEL codes: C73, H23, L13, O31, Q2, Q3

Keywords: environmental externality, resource extraction, oligopoly, Pigouvian taxation, R\&D

${ }^{*}$ We would like to thank Roberto Cellini and Arsen Palestini for useful comments and suggestions. The usual disclaimer applies. 


\section{Introduction}

The conflict between individual incentives and the preservation of the environment and natural resources, and the associated market failures, are well known since Gordon (1954) and Hardin's (1968) tragedy of the commons. In the subsequent decades, the economic literature has produced countless contributions concerning either the exploitation of natural resources or the environmental externalities generated by industrial activities, but rarely - if ever - both at the same time, although the interplay between growth and the environment and the sustainability of our economic system are both generally viewed as a circular model with feedback effects. ${ }^{1}$

This partial approach to a single side of the problem at a time is quite common in both static and dynamic applications of oligopoly theory to environmental or resource economics. Some recurrent themes emerging from this strand of literature can be quickly recollected so as to fix ideas. A cornerstone of the discussion is the market failure associated with external effects:

- Firms do not internalise environmental externalities, and therefore will not spontaneously invest in green technologies. This prompts the design of Pigouvian taxation to subminister the proper R\&D incentives to firms. ${ }^{2}$

- For analogous reasons, firms may overexploit natural resources, renewable or not. ${ }^{3}$

Another critical point concerns the pros and cons of any variation in industry output. Any industrial economist would agree that market power is detrimental to welfare because of high prices and low output levels. However, expanding output puts additional pressure upon the environment and the stock of natural resources. This reveals the presence of a tradeoff between the static efficiency usually associated to marginal cost pricing and the dynamic

\footnotetext{
${ }^{1}$ This view is so largely shared in the profession, that it appears regularly in the introductory chapters of textbook at any level (see, e.g., Pearce and Turner, 1989; Tisdell, 2009; and Anderson, 2010).

${ }^{2}$ See Downing and White (1986), Milliman and Prince (1989), Karp and Livernois (1994), Poyago-Theotoky (2007), inter alia.

${ }^{3}$ Classical contributions in this vein are those of Clark and Munro (1975) and Levhari and Mirman (1980). For a model of international trade with natural resource extraction, see Copeland and Taylor (2009), inter alia. An overview of the debate is in Long (2010).
} 
efficiency one should refer to in connection with the long-run sustainability of the current economic system.

Here we set out to revisit these issues through a differential game approach which, although being not properly a general equilibrium one, draws explicitly the endogenous link between the dynamics of resource exploitation and environmental externalities, to show that (i) pure profit incentives can indeed give rise to investments in green technologies which are ruled out in the conventional approach, and (ii) competition may actually exert positive long-run welfare effects, although with mixed feelings, as a cleaner environment is accompanied by a lower residual stock of natural resources. Our procedure will be the following. We will set out with the illustration of simple setups alternatively accounting for the presence of either natural resources or pollution, in open-loop games in which firms control output levels. Then, we will enrich the picture introducing a simple production function accounting for the fact that the natural resource enters the productive activities of firms as an input, and pollution may be subject to Pigouvian taxation which can be used as an incentive for green R\&D. Then, we will lay out a comprehensive model capturing the interplay between the output and R\&D decisions of firms on one side and the preservation of natural resources and the environment on the other. Whenever appropriated, we will also dwell on the optimal industry structure (i.e., the number of firms) in the commons, an issue that has received a considerable amount of attention in the early debate on common property in oligopoly (on this, see Cornes and Sandler, 1983; Cornes, Mason and Sandler, 1986; and Mason and Polasky, 1997, inter alia).

The remainder of the paper is structured as follows. In section 2, we offer a step-by-step reconstruction of the standard approach, whereby resource extraction and pollution are studied in isolation from each other. Then, in section 3, we propose a fully fledged model taking into account the interplay between state variables. Concluding remarks are in section 4 .

\section{Preliminaries: the standard approach}

Here we summarise the acquired wisdom based on previous literature, where either pollution or the exploitation of natural resources have been treated in isolation from one another. Throughout, we will consider an industrial sector existing over continuous time $t \in[0, \infty)$. 


\subsection{Natural resources I}

The simplest model of the interplay between profit incentives and resource extraction is the following. The market is supplied by $n$ firms offering a homogeneous good produced through a renewable natural resource (say, forestry) to deliver an intermediate or final commodity (say, timber or paper) to consumers. The market demand for the final good is

$$
p(t)=a-Q(t), Q(t)=\sum_{i=1}^{n} q_{i}(t),
$$

$q_{i}(t)$ being the instantaneous output of firm $i=1,2,3, \ldots n$. Therefore, the game features $n$ controls, $\mathbf{q}=\left(q_{1}, q_{2}, \ldots q_{n}\right)$, one for each player. All firms share a symmetric technology with the same marginal cost $c$ for extraction and production, giving rise to a cost function $C_{i}(t)=c q_{i}(t)$, with $a>c>0$. This imposes the constraint $Q(t) \in[0, a-c]$ at any time $t$. Additionally, market size $a-c$ is assumed to be large enough to ensure the non negativity of controls at all times during this and the subsequent versions of the game. The instantaneous profit function of firm $i$ is $\pi_{i}(t)=(p(t)-c) q_{i}(t)$.

The only state variable appearing in this version of the model is the stock of the natural resource $x(t) \geq 0$, which evolves over time according to the following state equation: ${ }^{4}$

$$
\dot{x}(t)=\eta x(t)-\sum_{i=1}^{n} q_{i}(t)
$$

where $\eta>0$ is the constant rate of reproduction.

The game is non cooperative and simultaneous play takes place at any instant. The individual firm has to

$$
\max _{q_{i}(t)} \int_{0}^{\infty} \pi_{i}(t) e^{-\rho t} d t
$$

${ }^{4}$ We could model the state equation as

$$
\dot{x}(t)=\eta x(t)-\nu \sum_{i=1}^{n} q_{i}(t)
$$

with $\nu \in(0,1]$. This, however, would not motify significantly the qualitative predictions of the our analysis. Therefore, we have imposed $\nu=1$ to restrict the set of parameters. 
subject to the dynamic constraints (2), the initial condition $x(0)=x_{0} \geq$ $n(a-c) /(n+1) / \eta,{ }^{5}$ and the appropriate transversality condition. The discount rate $\rho>0$ is constant and common to all firms.

Firm $i$ 's Hamiltonian function is

$\mathcal{H}_{i}(t)=e^{-\rho t}\left\{\left(a-q_{i}(t)-\sum_{j \neq i} q_{j}(t)-c\right) q_{i}(t)+\lambda_{i}(t)\left[\eta x(t)-\sum_{i=1}^{n} q_{i}(t)\right]\right\}$

in which $\lambda_{i}(t)=e^{\rho t} \gamma_{i}(t)$ is the co-state variable (in current value) associated with the dynamics of the state. This being a linear state game, the open-loop solution is subgame perfect (or equivalently, strongly time consistent).

The necessary conditions are ${ }^{6}$

$$
\begin{gathered}
\frac{\partial \mathcal{H}_{i}}{\partial q_{i}}=e^{-\rho t}\left(a-c-2 q_{i}-\sum_{j \neq i} q_{j}-\lambda_{i}\right)=0 \\
\dot{\lambda}_{i}=(\rho-\eta) \lambda_{i}
\end{gathered}
$$

together with the transversality conditions $\lim _{t \rightarrow \infty} \gamma_{i} x=\lim _{t \rightarrow \infty} e^{-\rho t} \lambda_{i} x=0$. Now observe that (6) admits the solution $\lambda_{i}=0$ at all times, whereby the first order condition on the output level yields the static Cournot-Nash solution $q_{i}=q^{N}=(a-c) /(n+1)$ for all $i$. Then, imposing stationarity on $\dot{x}$, one gets

$$
x^{N}=\frac{n q^{N}}{\eta}=\frac{n(a-c)}{(n+1) \eta}
$$

with $\partial x^{N} / \partial n>0$, which seems to indicate that increasing competition has positive consequences for the preservation of the natural resource in the long run.

The bottom line of this exercise is well known, as it states that firms replicate forever the equilibrium of the static Cournot game without internalising the effects of production on the existing amount of the natural resource. ${ }^{7}$

\footnotetext{
${ }^{5}$ Taking $x_{0}>0$ as the initial condition would not ensure the sustainability of extraction activities over $t \in[0, \infty)$ as the stock $x(t)$ would become nil in finite time.

${ }^{6}$ Henceforth, we omit the time argument for brevity. Mangasarian's (1966) and Arrow's (1968) sufficiency conditions are also satisfied. They are also omitted for brevity.

${ }^{7}$ This is the reason why we have taken the initial stock to be at least as large as the Cournot-Nash industry output.
} 
The industry output is therefore increasing with $n$, and this causes, at any time $t$, an increase in the rate of extraction as compared to pure monopoly, due to the output restriction that is usually associated with monopolistic sectors, as compared to any even slightly more competitive industries. On the other hand, any increase in output lowers market price and brings about an increase in consumer surplus $C S^{N}=Q^{N} / 2$. The balance between these effects is captured by the net effect of a change in $n$ on the social welfare function $^{8}$

$$
S W^{N}=n \pi^{N}+C S^{N}+x^{N}
$$

with

$$
\frac{\partial S W^{N}}{\partial n}=\frac{(a-c)[n+1+\eta(a-c)]}{\eta(n+1)^{3}}>0
$$

for all $n$. That is, competition is promoting social welfare, notwithstanding the fact that it involves a higher exploitation rate at any time. The next step consists in taking into consideration a slightly richer version of the same problem, which explicitly acknowledges the presence of an endogenous link between the natural resource and the output via a simple production function.

\section{$2.2 \quad$ Natural resources II}

Define now the individual output of firm $i$ as $q_{i}=b_{i} x, b_{i}$ being the instantaneous rate at which firm $i$ extract the resource and uses it in the production of the intermediate or final good. This can be thought of as a production function operating at constant returns to scale, using the natural resource as the only relevant input. We shall see in the remainder of the section that this seemingly simple transformation indeed has relevant consequences on our understanding and interpretation of the problem at hand. As in the previous version, we have $n$ controls, $\mathbf{b}=\left(b_{1}, b_{2}, \ldots b_{n}\right)$, one for each player,

\footnotetext{
${ }^{8}$ Note that the amount of natural resource enters the social welfare function with a weight equal to one, i.e., the same attached to industry profits and consumer surplus. The ongoing debate on this point hasn't yet produced a unanymous view (see, e.g., Stern, 2009 , ch. 5). The need of guaranteeing the prosperity of future generations suggest that one should attach to the preservation of natural resources at least the same importance as traditional economic indicators strictly related to production and consumption.
} 
while there is a single state, $x$, whose dynamics is now

$$
\dot{x}=\left(\eta-\sum_{i=1}^{n} b_{i}\right) x
$$

so that the insertion of a simple linear technology in the model establishes a multiplicative effect between state and control in the state equation which was altogether absent in the previous version. The remainder of the setup is unmodified, so the Hamiltonian of firm $i$ is

$$
\mathcal{H}_{i}=e^{-\rho t}\left[\left(a-b_{i} x-x \sum_{j \neq i} b_{j}-c\right) b_{i} x+\lambda_{i}\left(\eta-\sum_{i=1}^{n} b_{i}\right) x\right] .
$$

The game is thus no longer a linear state one, ${ }^{9}$ but for the sake of comparability we stick to the open-loop solution, requiring the following necessary conditions:

$$
\begin{gathered}
\frac{\partial \mathcal{H}_{i}}{\partial b_{i}}=e^{-\rho t} x\left(a-c-2 b_{i} x-x \sum_{j \neq i} b_{j}-\lambda_{i}\right)=0 \\
\dot{\lambda}_{i}=\left(\rho-\eta+\sum_{i=1}^{n} b_{i}\right) \lambda_{i}-b_{i}\left[a-c-2 x\left(\sum_{j \neq i} b_{j}+2 b_{i}\right)\right],
\end{gathered}
$$

while the transversality conditions are $\lim _{t \rightarrow \infty} e^{-\rho t} \lambda_{i} x=0$.

To simplify calculations, henceforth we impose symmetry on controls and co-states, $b_{i}=b_{j}=b$ and $\lambda_{i}=\lambda_{j}=\lambda$ for all $i, j$. From (12) we obtain

$$
b=\max \left\{\frac{a-c-\lambda}{(n+1) x}, 0\right\}
$$

and, if $b>0$,

$$
\lambda=a-c-b(n+1) x .
$$

Then, employing (10), we can write

$$
\dot{b}=-\frac{x \dot{\lambda}+(a-c-\lambda) \dot{x}}{(n+1) x^{2}}
$$

\footnotetext{
${ }^{9}$ Additionally, note that it is not defined in linear-quadratic form. Consequently, we have no obvious conjecture as to the form of the value function.
} 
which, using (13-15) can be rewritten as

$$
\dot{b}=\frac{(a-c)[\eta-\rho-b(n-1)]+b[\rho+(2 n b+\rho) n-2(n+1) \eta] x}{(n+1) x^{2}}
$$

Imposing stationarity on state and control, we identify the unique openloop Nash equilibrium, where: ${ }^{10}$

$$
b_{i}=b^{N}=\frac{\eta}{n} \forall i ; x^{N}=\frac{(a-c)(\eta-n \rho)}{\eta[2 \eta-(n+1) \rho]} .
$$

Note that $x^{N}>0$ for all

$$
\rho \in\left(0, \frac{\eta}{n}\right) \text { and } \rho>\frac{2 \eta}{n+1} .
$$

The same obviously holds for the individual output $q^{N}=b^{N} x^{N}$ as well as for the industry output $Q^{N}=n q^{N}$. Steady state individual profits are

$$
\pi^{N}=\frac{(a-c)^{2}(\eta-\rho)(\eta-n \rho)}{n[2 \eta-(n+1) \rho]^{2}}>0 \forall \rho \in\left(0, \frac{\eta}{n}\right) .
$$

Hence, the survival of firms at the steady state requires indeed $\rho \in(0, \eta / n)$. Now observe that

$$
\frac{\partial x^{N}}{\partial n}=\frac{(a-c)(\rho-\eta)}{\eta[2 \eta-(n+1) \rho]^{2}}<0
$$

for all $\rho \in(0, \eta)$. In view of the previous result, this is surely the case for any $n \geq 1$. This contradicts the result of the previous - and simpler - version of this problem, as now it appears that a more intense competition throughout the game leads to a lower amount of natural resource left over at the steady state.

Now reconsider the social welfare function, defined as in (8). Its partial derivative w.r.t. $n$ is

$$
\frac{\partial S W^{N}}{\partial n}=\frac{(a-c)(\eta-\rho)[\rho(n+1+\eta(a-c))-\eta(2+\eta(a-c))] \rho}{\eta[2 \eta-(n+1) \rho]^{3}}
$$

\footnotetext{
${ }^{10}$ The corresponding value of the co-state variable at the steady state Nash equilibrium is

$$
\lambda^{N}=\frac{(a-c)(n-1) \eta}{n[2 \eta-(n+1) \rho]}
$$

and the transversality condition $\lim _{t \rightarrow \infty} e^{-\rho t} \lambda_{i} x=0$ is met thanks to exponential discounting.
} 
which is positive iff

$$
\rho>\frac{\eta(2+\eta(a-c))}{n+1+\eta(a-c)}
$$

However,

$$
\frac{\eta(2+\eta(a-c))}{n+1+\eta(a-c)}-\frac{2 \eta}{n+1}=\frac{\eta^{2}(a-c)(n-1)}{[n+1+\eta(a-c)](n+1)}>0 \forall n \geq 2 .
$$

Hence, the equilibrium level of social welfare monotonically decreases in $n$ once the interplay between the natural resource and the firms' output has been duly accounted for, although admittedly in a very simple manner. ${ }^{11}$ Once again, the sign of the partial derivative (22) is reversed as compared to the previous setup. The foregoing discussion can be summarised in

Proposition 1 Endogenising the technological link between the exploitation of the natural resource and the intermediate or final output of the industry singles out the negative effect of an increase in the intensity of competition on the resulting equilibrium level of social welfare.

\subsection{Pollution I}

Let us now turn to an alternative scenario where any natural resources are left out of the picture, and the focus is on the environmental consequences of production (or consumption). Still, we consider the same $n$-firm oligopoly offering a homogeneous good, which now generates an undesirable environmental externality. Let $s(t) \geq 0$ be the stock of environmental pollution at any instant. We assume that pollution follows the dynamic equation:

$$
\dot{s}=z \sum_{i=1}^{n} q_{i}-\sum_{i=1}^{n} k_{i}-\delta s
$$

that is, it increases with the industry output level $Q=\sum_{i=1}^{n} q_{i}$ at a constant rate $z>0$, while it decreases with the industry's green $\mathrm{R} \& \mathrm{D}$ investments $K=\sum_{i=1}^{n} k_{i}, k_{i}$ being the instantaneous $\mathrm{R} \& \mathrm{D}$ effort of firm $i$ at the cost

\footnotetext{
${ }^{11}$ This result relates the long-run effects of increasing the population of firms with the discount rate. This aspect is a crucial feature of an ongoing debate concerning the need of applying low discount rates to the well-being of future generation (see Stern, 2007; and Weitzman, 2007, inter alia).
} 
$\Gamma_{i}=v k_{i}^{2}, v>0$. Pollution by itself diminishes at the constant rate $\delta \geq 0$. Hence, we are considering a single state, $s$ and $2 n$ controls, $\mathbf{q}=\left(q_{1}, q_{2}, \ldots q_{n}\right)$ and $\mathbf{k}=\left(k_{1}, k_{2}, \ldots k_{n}\right)$, two for each player.

The market demand function is $p=a-Q$, while firm i's production involves a cost function $C_{i}\left(q_{i}\right)=c q_{i}, c>0$. The Hamiltonian of firm $i$ is

$$
\mathcal{H}_{i}=e^{-\rho t}\left[\left(a-q_{i}-\sum_{j \neq i} q_{j}-c\right) q_{i}-v k_{i}^{2}+\mu_{i}\left(z \sum_{i=1}^{n} q_{i}-\sum_{i=1}^{n} k_{i}-\delta s\right)\right]
$$

where $\mu_{i}=e^{\rho t} \varpi_{i}$ is the co-state variable (in current value) associated with $s$. Strategic interaction is simultaneous, and we shall focus on the open-loop non cooperative Nash solution. This game is a linear state one, so that (i) the open-loop Nash equilibrium is subgame perfect, and (ii) we may anticipate that unregulated firms will never spontaneously internalise the environmental consequences of their productive activities at any time during the game. This ultimately implies that they will not invest in $\mathrm{R} \& \mathrm{D}$, as can be ascertained through a quick examination of the necessary conditions:

$$
\begin{gathered}
\frac{\partial \mathcal{H}_{i}}{\partial q_{i}}=e^{-\rho t}\left(a-c-2 q_{i}-\sum_{j \neq i} q_{j}-z \mu_{i}\right)=0 \\
\frac{\partial \mathcal{H}_{i}}{\partial k_{i}}=-e^{-\rho t}\left(2 v k_{i}+\mu_{i}\right)=0 \\
\dot{\mu}_{i}=(\delta+\rho) \mu_{i}
\end{gathered}
$$

Since (29) admits the solution $\mu_{i}=0$ at all times, ${ }^{12}$ this immediately entails that $k_{i}^{N}=0$ and $q_{i}^{N}=(a-c) /(n+1)$ at any time $t$. The transversality condition $\lim _{t \rightarrow \infty} \varpi_{i} s=\lim _{t \rightarrow \infty} e^{-\rho t} \mu_{i} s=0$ is also met. The resulting level of pollution at the steady state is $s^{N}=n(a-c) z /[(n+1) \delta]$, with $\partial s^{N} / \partial n>$ 0 .

\subsection{Pollution II}

As is well known, the way out of this impasse consists in introducing a Pigouvian taxation/subsidization $\mathcal{P}$ proportional to the stock of pollution,

\footnotetext{
${ }^{12}$ Note that $\mu_{i}=0$ suffices to ensure that the transversality condition $\lim _{t \rightarrow \infty} \mu_{i} s=0$ be satisfied.
} 
say, $\mathcal{P}=\theta s$, on all firms alike (see, e.g., Benchekroun and Long, 1998, 2002), $\theta$ being the tax or subsidy rate, which we will take to be constant throughout the game, for reasons that will be clarified below. ${ }^{13}$ This transforms the Hamiltonian of firm $i$ into the following:

$\mathcal{H}_{i}=e^{-\rho t}\left[\left(a-q_{i}-\sum_{j \neq i} q_{j}-c\right) q_{i}-v k_{i}^{2}-\theta s+\mu_{i}\left(z \sum_{i=1}^{n} q_{i}-\sum_{i=1}^{n} k_{i}-\delta s\right)\right]$.

As before, firms play simultaneously and non cooperatively at all times, taking now as given the Pigouvian policy set by the government. Observe that a direct consequence of the presence of regulation is that

$$
\dot{\mu}_{i}=(\delta+\rho) \mu_{i}+\theta
$$

which does not admit the nil solution any more and therefore opens the way for positive R\&D investments and also influences firms' output decisions:

$$
q_{i}=q^{N}=\frac{a-c-2 v z k^{N}}{n+1} ; k_{i}=k^{N}=\frac{\theta}{2(\delta+\rho) v} \forall i
$$

with $q^{N}>0$ provided $a-c>z \theta /(\delta+\rho)$. the optimal output $q^{N}$ is decreasing with $k^{N}$ and the optimal $\mathrm{R} \& \mathrm{D}$ effort $k^{N}$ is increasing with $\theta$, in such a way that - if firms are being taxed, i.e., for all $\theta>0$ - R\&D efforts are positive and the industry output is lower than in the unregulated case (the opposite holds of course if firms are subsidised, which happens for $\theta<0$ ). The resulting level of pollution at equilibrium is

$$
s^{N}=\frac{2 v(a-c)(\delta+\rho) z-\theta\left(n+1+2 v z^{2}\right)}{2 \delta(n+1)(\delta+\rho) v} .
$$

Then, the question arises as to how the government should choose the optimal $\theta$. Since $\theta$ appears in the firms' first order conditions, the openloop solution of the Stackelberg game with the government leading would obviously be subject to a problem of time inconsistency. To avoid it, one may either solve the Stackelberg game via a degenerate Markov approach (see

\footnotetext{
${ }^{13}$ Therefore, we have a single state, $s$ and $2 n+1$ controls, i.e., $\mathbf{q}=\left(q_{1}, q_{2}, \ldots q_{n}\right)$ and $\mathbf{k}=\left(k_{1}, k_{2}, \ldots k_{n}\right)$, two for each firm, and the Pigouvian policy rate $\theta$ for the government.
} 
Dockner et al., 2000, ch. 5), or simply calculate the value of $\theta$ maximising the steady state level of social welfare ${ }^{14}$ defined as

$$
S W^{N}=n \pi^{N}+C S^{N}-s^{N}(1-n \theta) .
$$

This requires solving

$$
\frac{\partial S W^{N}}{\partial \theta}=0
$$

yielding

$$
\theta^{*}=\frac{(\delta+\rho)\left[(n+1)\left(n+1+2 v z^{2}\right)-2 \delta(a-c) v z\right]}{\delta\left[n^{2}+1+2\left(1+v z^{2}\right) n\right]}
$$

which may take either positive or negative values depending on the relative size of parameters $\{a, c, n, v, z, \delta, \rho\}$, in particular $n$ and $a-c$. We have that

$$
\left.S W^{N}\right|_{\theta=\theta^{*}}>\left.S W^{N}\right|_{\theta=0}
$$

always, while

$$
\left.\operatorname{sign} s^{N}\right|_{\theta=\theta^{*}}-\left.s^{N}\right|_{\theta=0}=\operatorname{sign}\left[2 \delta(a-c) v z-(n+1)\left(n+1+2 v z^{2}\right)\right]
$$

which delivers the following:

Proposition 2 For all

$$
\begin{gathered}
a-c \in\left(0, \frac{(n+1)\left(n+1+2 v z^{2}\right)}{2 \delta v z}\right), \\
\begin{array}{c}
\text { For all }\left.\operatorname{and}(i) S W^{N}\right|_{\theta=\theta^{*}}>\left.S W^{N}\right|_{\theta=0} ;\left.(i i) s^{N}\right|_{\theta=\theta^{*}}<\left.s^{N}\right|_{\theta=0} \\
a-c>\frac{(n+1)\left(n+1+2 v z^{2}\right)}{2 \delta v z}
\end{array} \\
\theta<0 \text { and (i) }\left.S W^{N}\right|_{\theta=\theta^{*}}>\left.S W^{N}\right|_{\theta=0} ; \text { (ii) }\left.s^{N}\right|_{\theta=\theta^{*}}>\left.s^{N}\right|_{\theta=0} .
\end{gathered}
$$

The second claim appearing in the above Proposition states that, if market size is sufficiently large (either because the reservation price $a$ is high or because the marginal cost $c$ is low), the Pigouvian policy takes the form of a subsidy leading to a level of pollution higher than it would be without regulation. The increase in welfare, which obtains irrespective of whether firms

\footnotetext{
${ }^{14}$ This is the route taken by Benchekroun and Long (1998, 2002).
} 
are being taxed or subsidised, obtains because of the output expansion that is brought about by subsidization and ultimately increases consumer surplus. This is a direct consequence of the aforementioned tradeoff between the price effect and the external effect which, provided the market is affluent enough, paradoxically induces the regulator to opt for a higher consumer surplus even though this entails a larger amount of pollution. ${ }^{15}$

It can be shown that $\left.\partial S W^{N}\right|_{\theta=\theta^{*}} / \partial n>0$ always. ${ }^{16}$ The analysis of the effects of a change in $n$ on industry profits can only be carried out numerically, revealing that the industry concentration which maximises collective profits is decreasing in $\delta$. To see this, we fix $a-c=1, v=3, z=1$ and $\rho=1 / 5$, and solve $\left.\partial n \pi^{N}\right|_{\theta=\theta^{*}} / \partial n=0$ for different values of $\delta$, obtaining ( $n$ is rounded to the lower integer):

$$
\begin{aligned}
& n=58 \text { for } \delta=\frac{1}{5} \\
& n=73 \text { for } \delta=\frac{1}{4} \\
& n=99 \text { for } \delta=\frac{1}{3} \\
& n=149 \text { for } \delta=\frac{1}{2} \\
& n=224 \text { for } \delta=\frac{3}{4} \\
& n=239 \text { for } \delta=\frac{4}{5}
\end{aligned}
$$

That is,

Remark 3 The higher is the environment's degree of efficiency in recycling pollution, the larger is the population of firms maximising industry profits at

\footnotetext{
${ }^{15}$ It is worth noting that this mechanism would still exist in a simpler version of this setup, without R\&D investments. This is due to the fact that the conflict between two equally desirable objectives (lowering the price and reducing pollution) is entirely inherent to production decisions only.

${ }^{16}$ The proof, trivial but lengthy, is omitted for brevity. It is however available from the authors upon request.
} 
the steady state equilibrium in which a benevolent regulator adopts the socially optimal Pigouvian policy.

\section{The full model}

We are now ready to investigate a full-fledged model in which the natural resource enters explicitly in the production function of the intermediate or final output, and productive activities generate a negative environmental externality. To do so, we modify the state equations as follows.

We pose that pollution evolves according to the following equation:

$$
\dot{s}=z x \sum_{i=1}^{n} b_{i}-\sum_{i=1}^{n} k_{i}-\delta x,
$$

where the only detail that has changed as compared to the previous version is that the environment is being cleaned at a rate $\delta \geq 0$ by the existing amount of natural resource. ${ }^{17}$

The dynamics of the natural resource is

$$
\dot{x}=\left(\eta-\sum_{i=1}^{n} b_{i}\right) x-s,
$$

in which, note, the stock of pollution enters negatively.

All of the control variables have been already defined. Thus, the present game features two state variables, $s$ and $x$, and $2 n$ controls, $\mathbf{q}=\left(q_{1}, q_{2}, \ldots q_{n}\right)$ and $\mathbf{k}=\left(k_{1}, k_{2}, \ldots k_{n}\right)$, two for each player. We disregard the possibility of regulation through a Pigouvian policy for reasons that will become apparent below. The instantaneous profit function of firm $i$ is

$$
\pi_{i}=(p-c) b_{i} x-v k_{i}^{2}
$$

so that the individual firm must

$$
\max _{b_{i}, k_{i}} \int_{0}^{\infty} \pi_{i} e^{-\rho t} d t
$$

\footnotetext{
${ }^{17}$ This applies to rain forests and the oceans absorbing $\mathrm{CO}_{2}$ emissions, while it doesn't apply to other natural resources, like the stock of fish. On the contrary, the latter is negatively affected by pollution (as specified in (2), (10) and (41)).
} 
subject to the dynamic constraints (40-41), initial conditions $s(0)=s_{0}>$ 0 and $x(0)=x_{0}>s /\left(\eta-\sum_{i=1}^{n} b_{i}\right)$, and the appropriate transversality conditions. Once again, we solve the game under open-loop information. The firm's Hamiltonian function is

$$
\begin{gathered}
\mathcal{H}_{i}=e^{-\rho t}\left\{\left(a-b_{i} x-x \sum_{j \neq i} b_{i}-c\right) b_{i} x-v k_{i}^{2}\right. \\
\left.+\varphi_{i}\left(z x \sum_{i=1}^{n} b_{i}-\sum_{i=1}^{n} k_{i}-\delta x\right)+\psi_{i}\left[\left(\eta-\sum_{i=1}^{n} b_{i}\right) x-s\right]\right\},
\end{gathered}
$$

variables $\varphi_{i}=e^{\rho t} \zeta_{i}$ and $\psi_{i}=e^{\rho t} \varkappa_{i}$ being the co-states (in current value) associated with $s(t)$ and $x(t)$, respectively. The maximization of (44) requires meeting the following set of necessary conditions:

$$
\begin{gathered}
\frac{\partial \mathcal{H}_{i}}{\partial b_{i}}=e^{-\rho t} x\left(a-c-2 b_{i} x-x \sum_{j \neq i} b_{j}+z \varphi_{i}-\psi_{i}\right)=0 \\
\frac{\partial \mathcal{H}_{i}}{\partial k_{i}}=-e^{-\rho t}\left(2 v k_{i}+\varphi_{i}\right)=0 \\
\dot{\varphi}_{i}=\rho \varphi_{i}+\psi_{i} \\
\dot{\psi}=\left(b_{i}+\rho-\eta-\sum_{j \neq i} b_{j}\right) \psi+\left(\delta-z b_{i}\right) \varphi_{i}-b_{i}\left(a-c-x\left(2 b_{i}+\sum_{j \neq i} b_{j}\right)\right) .
\end{gathered}
$$

The associated transversality conditions are

$$
\lim _{t \rightarrow \infty} e^{-\rho t} \varphi_{i} s=0 ; \lim _{t \rightarrow \infty} e^{-\rho t} \psi_{i} x=0 \forall i .
$$

From (45), we obtain

$$
\psi_{i}=a-c-2 b_{i} x-x \sum_{j \neq i} b_{j}+z \varphi_{i}
$$

and

$$
\dot{b}_{i}=\frac{x\left(z \dot{\varphi}_{i}-\dot{\psi}_{i}-x \sum_{j \neq i} \dot{b}_{j}\right)-\left(a-c+z \varphi_{i}-\psi_{i}\right) \dot{x}}{2 x^{2}}
$$


while from (46) we get

$$
\varphi_{i}=-2 b k_{i}
$$

and therefore also

$$
\dot{k}_{i}=-\frac{\dot{\varphi}_{i}}{2 b}=-\frac{\rho \varphi_{i}+\psi_{i}}{2 b} .
$$

Before proceeding any further, it is worth noting that (53) implies:

Lemma 4 The interplay between state variables allows for the presence of positive REDD efforts during the game.

Substituting (47), (48), (50) and (52) into (51) and (53), and imposing symmetry across firms, we can write the control equations as follows:

$$
\begin{gathered}
\dot{b}=\{(a-c)[\eta-\rho+z-b(n-1)]+2 v k[\delta+z(b(n-1)-\eta-z)]+ \\
b(n+1)[s-x(b-\rho+z-2(n b-\eta))]\} /[(n+1) x] \\
\dot{k}=\frac{b(n+1) x+2 v(\rho+z) k-a+c}{2 v}
\end{gathered}
$$

This version of the control equations reveals a relevant property of the game, namely that, at any time,

$$
\dot{k}>0 \text { if } a-c<b(n+1) x+2 v(\rho+z) k .
$$

In other words, this condition says that the individual $R \& D$ effort in green technologies will increase provided market size is 'small'. An equivalent reading is that R\&D efforts will increase if the population of firms is large enough. Either way, it boils down to saying that the pace of green innovation is positively related to the intensity of competition characterising this industry. ${ }^{18}$

Imposing stationarity on the system $\{\dot{x}, \dot{s}, \dot{b}, \dot{k}\}$, we obtain the coordinates of the unique Nash equilibrium of the open-loop game:

$$
x^{N}=\frac{n(a-c) z}{(n+1) \delta} ; s^{N}=\frac{n(a-c)(\eta z-\delta)}{(n+1) \delta} ; b^{N}=\frac{\delta}{n z} ; k^{N}=0 .
$$

\footnotetext{
${ }^{18}$ This result has a definite Arrovian flavour. For a summary of the debate between Schumpeter (1942) and Arrow (1962) on the relationship between market power and innovation incentives, see, e.g., Tirole (1988).
} 
This steady state obviously coincides with the monopoly equilibrium if $n=1$, and it is admissible provided that $\eta \geq \delta / z$. Steady state output and profits are $q^{N}=(a-c) /(n+1)$ and $\pi^{N}=(a-c)^{2} /(n+1)^{2}$, i.e., the standard Cournot-Nash profits, and $\varphi=\psi=0$.

Now we can examine the steady state social welfare level, defined as

$$
\begin{gathered}
S W^{N}=n \pi^{N}+C S^{N}+x^{N}-s^{N}= \\
\frac{n(a-c)[(2(a-c+1)+n(a-c+2)) \delta+2(n+1)(1-\eta) z]}{2 \delta(n+1)^{2}} .
\end{gathered}
$$

The effect of a change in $n$ on welfare is described by

$$
\frac{\partial S W^{N}}{\partial n}=\frac{(a-c)[\delta(a-c+n+1)+(n+1)(1-\eta) z]}{\delta(n+1)^{3}}
$$

$\eta \in(0,1]$ being a sufficient condition for $\partial S W^{N} / \partial n>0$ for all $n$. In this region (i.e., if the instantaneous regeneration rate of the natural resource is less than $100 \%$, which, realistically, will almost always be the case), any increase in the intensity of competition generated by an increase in the population of firms is indeed beneficial. More precisely, the overall effect of a change in $n$ on $S W^{N}$ can be decomposed as follows:

$$
\frac{\partial S W^{N}}{\partial n}=\frac{\partial\left(n \pi^{N}\right)}{\partial n}+\frac{\partial\left(C S^{N}\right)}{\partial n}+\frac{\partial x^{N}}{\partial n}-\frac{\partial s^{N}}{\partial n}
$$

with

$$
\frac{\partial\left(n \pi^{N}\right)}{\partial n}<0 ; \frac{\partial\left(C S^{N}\right)}{\partial n}>0 ; \frac{\partial x^{N}}{\partial n}>0 ; \frac{\partial s^{N}}{\partial n}<0,
$$

whereby it appears that the negative effect on industry profits is more than compensated by the increase in consumer surplus, the higher volume of the natural resource and the lower level of pollution. Also, note the opposite sign of the partial derivatives of $s^{N}$ and $x^{N}$ w.r.t. $n$ : a cleaner environment goes along with a higher exploitation of the natural resource in steady state, the larger the population of firms is.

If instead $\eta>1, \partial S W^{N} / \partial n=0$ in

$$
n^{*}=\frac{(\eta-1) z-(a-c+1) \delta}{\delta-(\eta-1) z}>1
$$


for all

$$
\delta \in\left(\frac{2(\eta-1) z}{a-c+2},(\eta-1) z\right) .
$$

This discussion can be summarised in

Proposition 5 If $\eta \in(0,1]$, then any increase in the intensity of competition exerts a welfare-increasing effect. If instead $\eta>1$, then the socially optimal number of firms is finite, monopoly being Pareto-efficient for all $\delta$ outside the interval

$$
\left(\frac{2(\eta-1) z}{a-c+2},(\eta-1) z\right) .
$$

To ascertain the stability properties of the system, one has to inspect the following Jacobian matrix:

$$
J=\left[\begin{array}{llll}
\frac{\partial \dot{x}}{\partial x} & \frac{\partial \dot{x}}{\partial s} & \frac{\partial \dot{x}}{\partial b} & \frac{\partial \dot{x}}{\partial k} \\
\frac{\partial \dot{s}}{\partial x} & \frac{\partial \dot{s}}{\partial s} & \frac{\partial \dot{s}}{\partial b} & \frac{\partial \dot{s}}{\partial k} \\
\frac{\partial \dot{b}}{\partial x} & \frac{\partial \dot{b}}{\partial s} & \frac{\partial \dot{b}}{\partial b} & \frac{\partial \dot{b}}{\partial k} \\
\frac{\partial \dot{k}}{\partial x} & \frac{\partial \dot{k}}{\partial s} & \frac{\partial \dot{k}}{\partial b} & \frac{\partial \dot{k}}{\partial k}
\end{array}\right]
$$

whose eigenvalues can be easily calculated in the special case of a single firm (i.e., $n=1$ ), with

$$
\begin{gathered}
\frac{\partial \dot{x}}{\partial x}=\eta-b ; \frac{\partial \dot{x}}{\partial s}=-1 ; \frac{\partial \dot{x}}{\partial b}=-x ; \frac{\partial \dot{x}}{\partial k}=0 \\
\frac{\partial \dot{s}}{\partial x}=z b-\delta ; \frac{\partial \dot{s}}{\partial s}=0 ; \frac{\partial \dot{s}}{\partial b}=z x ; \frac{\partial \dot{s}}{\partial k}=-1 \\
\frac{\partial \dot{b}}{\partial x}=\frac{2[v(z(\eta+z)-v) k-b s]-(a-c)(\eta-\rho+z)}{2 x^{2}} ; \frac{\partial \dot{b}}{\partial s}=\frac{b}{x} \\
\frac{\partial \dot{b}}{\partial b}=\frac{s+x[2(b+\eta)+\rho-z]}{x} ; \frac{\partial \dot{b}}{\partial k}=\frac{v[\delta-z(\eta+z)]}{x}
\end{gathered}
$$




$$
\frac{\partial \dot{k}}{\partial x}=\frac{b}{v} ; \frac{\partial \dot{k}}{\partial s}=0 ; \frac{\partial \dot{k}}{\partial b}=\frac{x}{v} ; \frac{\partial \dot{k}}{\partial k}=\rho+z .
$$

The resulting eigenvalues of $J$ in $\left\{x^{*}, s^{*}, b^{*}, k^{*}\right\}$ are:

$$
\begin{aligned}
& \varepsilon_{1}=\frac{\eta+\sqrt{\eta^{2}+4 \delta}}{2}>0 ; \varepsilon_{2}=\frac{\eta-\sqrt{\eta^{2}+4 \delta}}{2}<0 \\
& \varepsilon_{3}=\frac{2 \rho-\eta+\sqrt{\eta^{2}+4 \delta}}{2}>0 \\
& \varepsilon_{4}=-\frac{\eta-2 \rho+\sqrt{\eta^{2}+4 \delta}}{2}<0 \forall \rho<\frac{\eta+\sqrt{\eta^{2}+4 \delta}}{2} .
\end{aligned}
$$

Accordingly, $\left\{x^{*}, s^{*}, b^{*}, k^{*}\right\}$ is a saddle point equilibrium in the monopoly case. Performing the same analysis in the general case of an oligopoly is, however, cumbersome. Yet, we can work out some numerical examples. For instance, one can fix

$$
a-c=1 ; n=2 ; v=1 ; z=1 ; \delta=\frac{1}{30} ; \eta=\frac{1}{10} ; \rho=\frac{1}{100}
$$

to obtain

$$
\varepsilon_{1}=0.2725 ; \varepsilon_{2}=-0.2524 ; \varepsilon_{3}=0.0217 ; \varepsilon_{4}=-0.0164
$$

which again reveals saddle point stability - in this case, of the duopoly equilibrium.

\section{Concluding remarks}

We have modelled the dynamic interplay between firms' decisions and the resulting welfare performance of an industry involving the exploitation of a natural resource and negative environmental effects. Towards this aim, we have adopted a stepwise procedure, starting from the simplest settings to end up with a complete model including all relevant variables. This has been done with the purpose of illustrating how some of the main properties and policy conclusions may change depending upon the degree of accuracy and completeness which the model itself is endowed with. In particular, we have focussed on the tradeoff between the opposite effects of output expansions on market price on one side and the intensity of resource exploitation and 
environmental externality on the other. In this regard, a key aspect one has to bear in mind is the intensity of market competition, measured by the size of the population of active firms in the industry. The full-fledged model we have constructed indicates that, for any realistic rate of reproduction of the natural resource, any increase in the population of firms is indeed welfare-improving. A related issue we have also dwelled upon is whether firms may have any incentive to invest in green technologies in the absence of taxation/subsidization. In this respect, our model suggests that such an incentive does exist, due to competitive pressure, even if firms do not explicitly internalise the effects of their activities. 


\section{References}

[1] Anderson, D. (2010), Environmental Economics and Natural Resource Management. Third Edition, London, Routledge.

[2] Arrow, K.J. (1962), "Economic welfare and the allocation of resources for invention", in Nelson, R. (ed.), The Rate and Direction of Industrial Activity, Princeton, NJ, Princeton University Press.

[3] Arrow, K.J. (1968), "Applications of control theory to economic growth", in Dantzig, G.B. and A.F. Veinott (eds.), Mathematics of the Decision Sciences Part 2, Providence, American Mathematical Society.

[4] Benchekroun, H. and N.V. Long (1998), "Efficiency inducing taxation for polluting oligopolists", Journal of Public Economics, 70, 325-42.

[5] Benchekroun, H. and N.V. Long (2002), "On the multiplicity of efficiency-inducing tax rules", Economics Letters, 76, 331-336.

[6] Clark, C.W. and G.R. Munro (1975), "The economics of fishing and modern capital theory", Journal of Environmental Economics and Management, 2, 92-106.

[7] Copeland, B.R. and S.M. Taylor (2009), "Trade, tragedy, and the commons", American Economic Review, 99, 725-49.

[8] Cornes, R. and T. Sandler (1983), "On commons and tragedies", American Economic Review, 73, 787-92.

[9] Cornes, R., C.F. Mason and T. Sandler (1986), "The commons and the optimal number of firms", Quarterly Journal of Economics, 101, 641-46.

[10] Dockner, E.J, S. Jørgensen, N.V. Long and G. Sorger (2000), Differential Games in Economics and Management Science, Cambridge, Cambridge University Press.

[11] Downing, P.B. and L.J. White (1986), "Innovation in pollution control", Journal of Environmental Economics and Management, 8, 225-71.

[12] Gordon, H.S. (1954), "The economic theory of a common-property resource: the fishery", Journal of Political Economy, 62, 124-42. 
[13] Hardin, G. (1968), "The Tragedy of the Commons", Science, 162, 124348.

[14] Karp, L. and J. Livernois (1994), "Using automatic tax changes to control pollution emissions", Journal of Environmental Economics and Management, 27, 38-48.

[15] Levhari, D. and L. Mirman (1980), "The great fish war: An example using a dynamic Cournot-Nash solution", Bell Journal of Economics, 11, 322-34.

[16] Long, N.V. (2010), A Survey of Dynamic Games in Economics, Singapore, World Scientific, Singapore.

[17] Mangasarian, O.L. (1966), "Sufficient conditions for the optimal control of nonlinear systems", SIAM Journal on Control, 4, 139-52.

[18] Mason, C.F. and S. Polasky (1997), "The optimal number of firms in the commons: A dynamic approach", Canadian Journal of Economics, 30, $1143-60$.

[19] Milliman, S.R. and R. Prince (1989), "Firm incentives to promote technological change in pollution control", Journal of Environmental Economics and Management, 17, 247-65.

[20] Pearce, D.W. and R.K. Turner (1989), The Economics of Natural Resources and the Environment, Hemel Hempstead, Harvester Wheatsheaf.

[21] Poyago-Theotoky, J.A. (2007), "The organization of R\&D and environmental policy", Journal of Economic Behavior and Organization, 62, 63-75.

[22] Schumpeter, J.A. (1942), Capitalism, Socialism and Democracy, New York, Harper \& Row.

[23] Stern, N. (2007), The Economics of Climate Change: The Stern Review, Cambridge, Cambridge University Press.

[24] Stern, N. (2009), A Blueprint for a Safer Planet: How to Manage Climate Change and Create a New Era of Progress and Prosperity, London, Bodley Head. 
[25] Tirole, J. (1988), The Theory of Industrial Organization, Cambridge, MA, MIT Press.

[26] Tisdell, C. (2009), Resource and Environmental Economics. Modern Issues and Applications, Singapore, World Scientific.

[27] Weitzman, M.L. (2007), "A review of the Stern Review on the economics of climate change", Journal of Economic Literature, 45, 703-24. 


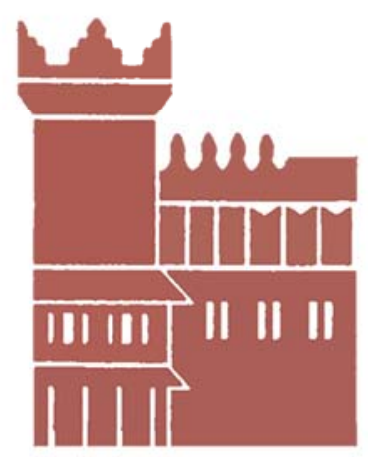

Alma Mater Studiorum - Università di Bologna DEPARTMENT OF ECONOMICS

Strada Maggiore 45

40125 Bologna - Italy

Tel. +39051 2092604

Fax +390512092664

http://www.dse.unibo.it 